Orientación familiar y hábitos saludables en estudiantes de Educación... Inés María Muñoz et al.

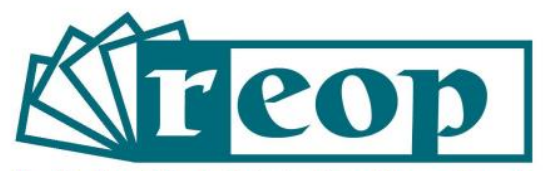

Revista Española de Orientación y Psicopedagogía

\title{
ORIENTACIÓN FAMILIAR Y HÁBITOS SALUDABLES EN ESTUDIANTES DE EDUCACIÓN PRIMARIA
}

\section{FAMILY GUIDANCE AND HEALTHY HABITS IN PRIMARY EDUCATION STUDENTS}

\author{
Inés $M^{\mathrm{a}}$ Muñoz-Galiano \\ Universidad de Jaén. Facultad de Humanidades y Ciencias de la Educación. Departamento de \\ Pedagogía. Jaén, España \\ Cristina Pinto Díaz \\ Universidad de Jaén. Facultad de Humanidades y Ciencias de la Educación. Jaén, España \\ Gema Torres-Luque ${ }^{1}$ \\ Universidad de Jaén. Facultad de Humanidades y Ciencias de la Educación. Departamento de \\ Didáctica de la Expresión Musical, Plástica y Corporal. Jaén, España
}

\section{RESUMEN}

La familia constituye un contexto socializador y educativo de sus miembros a lo largo del ciclo vital, especialmente para sus hijos, afectando a su desarrollo integral. En este sentido, es necesario identificar factores familiares que influyan en el proceso educativo a fin de perfilar las actuaciones desde la orientación familiar. El objetivo de este trabajo es conocer si el nivel educativo de los progenitores influye en el volumen de práctica de actividad física que realizan sus hijos/as estudiantes de Educación Primaria. Para ello, se seleccionaron 284 niños/as (6 a 11 años). A los padres/madres/tutores se les administró un cuestionario para conocer su nivel educativo (bajo, medio, alto) y los hábitos de actividad física que realizan sus hijos/as (toda la semana, entre semana, fin de semana). Los resultados indican que los hijos de las familias con un nivel educativo medio-alto cumplen con las recomendaciones de actividad física saludable, siendo a su vez volúmenes de actividad física estadísticamente significativos respecto a las familias con bajo nivel educativo. A su vez, los progenitores con diferente nivel educativo tienen una influencia

${ }^{1}$ Correspondencia: Gema Torres-Luque. Correo-e: gtluque@ujaen.es 
en una mayor práctica de actividad física respecto a los que lo tienen igual. Estos datos, ponen en relieve la importancia de orientar a las familias, especialmente en aquellas con bajo nivel educativo, en las capacidades y habilidades necesarias para promocionar hábitos saludables en el contexto familiar. Igualmente, los resultados son de interés para los centros educativos a fin de implicar a las familias en programas de promoción de la actividad física.

Palabras clave: orientación familiar, hábitos saludables, actividad física, educación, niños/as.

\begin{abstract}
The family is part of children's social and educational context. It is important to identify family factors that influence the educational process to outline actions for family guidance. From this perspective, the aim of this study is to know if the parents' educational level influences the amount of practice of physical activity carried out by their children in Primary Education. 284 children (6 to 11 years old) were selected. Their parents were asked about their educational level (low, medium, high) and about the physical activity of their children (throughout the week, school days and weekends). The results indicate that the children of families with a medium-high educational level comply with the recommendations for healthy physical activity. Also, they present higher amounts of physical activity at different times as compared to children from families with low educational levels. In turn, parents with different educational levels, have an influence on a greater practice of physical activity than those with the same level. These data highlight the importance of guiding families, especially those with a low educational level, on regarding the skills and abilities necessary to promote healthy habits in the family context. Likewise, the results are of interest to schools to involve families in programs to promote physical activity.
\end{abstract}

Key Words: family guidance, healthy habits, physical activity, education, children.

\title{
Cómo citar este artículo:
}

Muñoz-Galiano, I.M., Pinto Díaz, C. y Torres-Luque, G. (2021). Orientación familiar y hábitos saludables en estudiantes de educación primaria. Revista Española de Orientación y Psicopedagogía, 32(3), 60-73. https://doi.org/10.5944/reop.vol.32.num.3.2021.32556

\section{Introducción}

Los cambios sociales repercuten en el estilo de vida, convirtiéndose en ocasiones en factores de riesgo para la salud, especialmente en edad escolar (Dumith et al., 2011; Määttä et al., 2017). En este sentido, la infancia es una etapa decisiva en el aprendizaje y la consolidación de hábitos que tienden a mantenerse en el tiempo (Castillo et al., 2018; Hayes, 2017). La calidad de vida y la salud en la edad adulta dependen de hábitos adoptados desde los primeros años de vida (Bagordo et al., 2017). Ante esta situación, se precisan actuaciones desde los entornos educativos más influyentes: familia y escuela. Estos dos contextos, son claves en el desarrollo de una 
educación integral y de calidad, pues constituyen los espacios donde los niños reciben los primeros mensajes educativos (Cano y Casado, 2015). Desde esta perspectiva, las prácticas de orientación educativa han de estar presentes en ellos, y de modo especial en edades tempranas. Son diversos los motivos por los que, en ocasiones, requieren ayuda de profesionales externos que orienten en el proceso educativo. En este sentido, el apoyo desde la orientación familiar es un aspecto fundamental para poder promover el compromiso e iniciativa de la comunidad educativa, especialmente de la familia hacia prácticas de vida saludable (Torres et al., 2019).

La familia proporciona las pautas básicas de socialización a los miembros más jóvenes y promueve determinados valores, todo en el marco de la cultura, creencias y estilos de vida asumidos por cada grupo familiar en concreto (Álvarez y Bisquerra, 2012). En este contexto, comienza a construirse la persona y la educación familiar afectará a todos los ámbitos de cada uno de sus miembros. La familia a partir del cumplimiento de sus funciones constituye el sistema funcional formativo, que garantiza que el niño sobreviva y se desarrolle de la mejor forma posible. Su principal función se centra en la satisfacción de importantes necesidades, al tiempo que se produce la formación y transformación de la personalidad de sus integrantes, desarrollando hábitos y convicciones, al tiempo que definen los cimientos del comportamiento (Ramos y González, 2017).

La constancia e insistencia de la familia es fundamental para inculcar al proceso educativo de sus hijos, unos valores que promuevan un desarrollo integral y sigan unos estilos de vida saludables (Montesinos, 2014). No se puede olvidar que los hijos en edades tempranas aprenden por las conductas, actitudes y valores que observan y viven principalmente en el seno familiar. A lo que habría que añadir lo trabajado en el contexto escolar y el trabajo realizado entre ambos.

Existen evidencias de que la implicación de la familia en el proceso formativo de sus hijos/as y su cercanía al centro escolar, abogan por una mejora en su desarrollo personal y social (González, 2014), en el rendimiento académico, evitando el fracaso escolar (Parra et al., 2017; Sheldon y Epstein, 2002) y en sus actitudes y conductas (Siles-Rojas, 2003). En este sentido, son muchas las investigaciones que ponen de manifiesto la importancia y trascendencia de establecer buenas relaciones entre la familia y la escuela (Bernad y Llevot, 2015; Cano y Casado, 2015). Aún así, es cierto que, el acompañamiento de las familias a lo largo de la etapa de escolarización va disminuyendo, siendo menor en Educación Primaria y Secundaria respecto a Educación Infantil (Gomáriz et al., 2008). No obstante, la influencia que posee es elevada, y su presencia juega un papel fundamental en diferentes contextos, entre los que destaca hacer los centros educativos más eficaces (Azpillaga et al., 2014; Martínez-González et al., 2010). Así, la colaboración en la educación de los menores entre los centros educativos y la familia es fundamental, a pesar de los inconvenientes contextuales a salvar (Acuña-Collado, 2016), donde el trabajo conjunto se hace más necesario. Si bien, la responsabilidad en el desarrollo de hábitos a edades tempranas corresponde a la familia, se debe considerar que cada familia lo afronta de modo distinto. Lo cual, pone de relevancia la necesidad de profesionales que ofrezcan orientación al respecto (PérezBóveda y Yáñiz, 2015). Es decir, se precisa de ayuda que facilite una dinámica familiar positiva. En este sentido, la orientación educativa, y en concreto la orientación familiar, al comprender el estudio de la familia como contexto de socialización y aprendizaje de sus miembros a lo largo del ciclo vital, se constituye el marco desde el que actuar (Aguirre, 2015).

La familia influye directamente en el aprendizaje de sus hijos/as, en relación con diferentes factores, descritos como factores estructurales y no-estructurales (Cornejo y Redondo, 2007; Parra et al., 2017). Así, los primeros tienen relación con el nivel socioeconómico y educativo de los padres/madres, alimentación y salud o hábitos de lectura de los progenitores; siendo los segundos, factores relacionados con el clima afectivo, prácticas sociales, etc.

De esta manera, se ha observado como el entorno socioeconómico (Pàmies-Rovira et al., 2016) o el nivel de estudios de las familias (Fajardo-Bullón et al., 2017) son factores que condicionan el proceso de aprendizaje y, en mayor medida, el logro de los resultados académicos. Así mismo, se viene constatando cómo el entorno familiar y las características sociodemográficas 
que lo definen influyen en el desarrollo de hábitos sedentarios, el uso de los recursos electrónicos o de dispositivos móviles y se requiere de la orientación familiar para hacer frente a estos desafíos (Nogueira y Ceinos, 2015; Ramírez-García et al., 2020; Sousa y Silva, 2017; Wu et al., 2018). Igual importancia tiene la influencia de la familia en otros aspectos como pueden ser los relacionados con hábitos saludables, entre los que destaca, la actividad física (Edwardson y Gorely, 2010; Muñoz-Galiano et al., 2020; Muñoz-Galiano et al., 2019).

Sin lugar a duda, la actividad física es hoy día uno de los hábitos saludables para tener en cuenta en el desarrollo del niño/a. Poseer una mayor práctica de actividad física (AF), está asociado con un menor riesgo de padecer enfermedades en la etapa adulta (Bagordo et al., 2017), incrementa el autoconcepto (De la Torre-Cruz et al., 2015), la autoestima (Biddle y Asare, 2011) o incrementa el rendimiento académico (Ruiz-Ariza et al., 2016). La familia es un factor que ejerce influencia en este hecho, así, aspectos como el nivel socioeconómico (Bagordo et al., 2017; Drenowatz et al., 2010), ocupación fundamentalmente de la figura de la madre (Jalali-Farahani et al., 2017) o el nivel educativo (Määttä et al., 2017), son agentes condicionantes. De hecho, en edades entre los 6 y 11 años, aspectos como implicación de los progenitores, ánimo a sus hijos/as, etc., son aspectos que colaboran a practicar más actividad física (Edwarson y Gorely, 2010).

En este sentido, una de las variables que puede influir en una mayor o menor práctica de AF es el nivel de formación de los padres/madres. Sin embargo, existe cierta controversia en la literatura, ya que algunas indican que este hecho tiene influencia (Cuervo et al., 2017), frente a otras investigaciones que indican que no tiene por qué ser un factor principal (Cheung, 2017; Torrijos et al., 2015). Es cierto, que la multitud de estudios se llevan a cabo en poblaciones adolescentes, y son escasos los estudios en etapas educativas más tempranas, lo que podría dar lugar a dicha controversia. Por este motivo, es necesario seguir profundizando en la influencia que ejerce la familia, en este caso respecto a su nivel educativo, sobre un hábito saludable como es la mayor práctica de AF.

Conocer los indicadores que influyen en el desarrollo de hábitos saludables, y en concreto de la realización de actividad física, en estudiantes de Educación Primaria puede ayudar a planificar la orientación educativa y familiar. Desde este planteamiento, las implicaciones del estudio para dicho proceso se concretan entre otras, en un mayor conocimiento del contexto de intervención: "el enfoque ecológico reclama el análisis de los sistemas contextuales y de las relaciones que se establecen entre ellos, para llegar a una comprensión del funcionamiento y desarrollo de las personas y de los grupos de interacción" (Álvarez y Bisquerra, 2012, p. 410). Además, supone el reconocimiento de contextos en los que la orientación familiar se hace más necesaria. Si se confirma que el nivel educativo de la familia influye en el desarrollo de la actividad física de sus hijos, habría que actuar con aquellas familias con menor nivel de formación para paliar los efectos negativos que pudiera tener esta variable, en este caso, en el desarrollo de hábitos y la concienciación sobre la importancia de una vida saludable. Así mismo, desde la orientación habría que promover la colaboración entre la familia y el centro educativo a fin de actuar de forma coordinada y en consonancia con las demandas realizadas (Silvero, 2015).

Por lo tanto, el objetivo de este estudio es conocer si el nivel educativo de los progenitores influye en el volumen de práctica de actividad física semanal que realizan sus hijos/as estudiantes de Educación Primaria. No se puede olvidar que estas actitudes y patrones de conducta reciben una fuerte influencia del entorno familiar y la escuela, por lo que implicarán un reto para la orientación educativa y familiar (Romero-Blanco et al., 2020; Sawa et al., 2020; Torres et al., 2019). En este sentido, los datos permitirán establecer programas de orientación familiar y procesos de ayuda con el fin de facilitar el desarrollo de hábitos saludables desde el contexto familiar. Si las familias perciben la necesidad de realizar actividad física, así como la trascendencia de sus funciones como padres y madres y su repercusión en el desarrollo de actitudes en sus hijos, se podrá garantizar una respuesta adecuada a esta exigencia de la sociedad actual. 


\section{Método}

\section{Muestra}

La muestra estuvo compuesta por 284 estudiantes de Educación Primaria, con edades comprendidas entre los 6 y 11 años (131 chicos y 153 chicas). Todos ellos pertenecían a un colegio de entorno rural, con menos de diez mil habitantes (Chillón et al., 2011). La elección del centro fue intencional por proximidad. Participaron todos aquellos estudiantes de esta etapa educativa y que no tuviesen ningún problema que impidiese la práctica de actividad física. La muestra fue del $87 \%$ del $100 \%$ posible para ese centro educativo, donde un $10 \%$ no participaron por no completar los instrumentos seleccionados en su totalidad y, un 3\% por no realizar práctica de actividad física, por lesión o por poseer alguna patología. Los padres/madres/tutores de los estudiantes firmaron un consentimiento informado por escrito y el centro dio su autorización para llevar a cabo el estudio en sus instalaciones. Este trabajo está aprobado por el Comité de Ética de la institución local.

\section{Instrumentos y/o técnicas}

Para la recogida de información, se contactó con el centro escolar, se obtuvo un permiso de la dirección del mismos. A su vez, se realizó una reunión informativa para los padres/madres y se obtuvo un consentimiento informado por escrito. De esta forma, posteriormente en una sala habilitada para tal efecto se administraron dos cuestionarios utilizados en anteriores estudios, con preguntas relevantes para el objeto de estudio a pesar de no tener una autoría única. El primer cuestionario hace referencia al nivel educativo de los padres/madres/tutores y el segundo a la práctica de actividad física.

\section{Nivel educativo de los padres/madres/tutores}

A este colectivo se le preguntó por el grado de educación más alto que poseían. Se les facilitó las distintas opciones que se contemplan en la educación española. Las opciones de respuesta se categorizaron en tres niveles: a) Bajo nivel: sin graduación, Primaria/EGB, Secundaria/ESO; b) Medio nivel: Formación Profesional I, Ciclos formativos de grado medio, Bachillerato/BUP/COU, Formación Profesional II, Ciclo formativo de grado superior; y, c) Alto nivel: Diplomatura universitaria o ingeniería técnica, Licenciatura o ingeniería superior, Graduado, Máster, Doctorado. Este cuestionario ha sido empleado previamente en diferentes estudios (Cuervo et al., 2017; Määttä et al., 2017; Muñoz-Galiano et al., 2020). A su vez, se contempló también dos opciones más: a) Padre y Madre con el mismo nivel educativo y b) Padre y Madre con diferente nivel educativo; excluyendo de este análisis a aquellas familias monoparentales. Este aspecto también ha sido previamente considerado (Muñoz-Galiano et al., 2019). 


\section{Practica de actividad física}

El volumen de práctica de actividad física semanal se obtuvo preguntando sobre las diferentes opciones de llevarla a cabo. Las mismas contabilizaban en distintos apartados: con un carácter cualitativo a) Sobre desplazamiento activo: una pregunta cualitativa sobre el modo de desplazarse al centro escolar, 1= modo de ida (andando, en bicicleta, coche, moto, autobús) y, $2=$ modo de vuelta (andando, en bicicleta, coche, moto, autobús); que incluía a su vez una pregunta cuantitativa para conocer el volumen en minutos en que se cubre la ida y la vuelta del domicilio al centro escolar (<10 min, entre 10-15 min, entre 15-20 min, entre 20-30 min, >30 min), donde para el cómputo de AF total, solo se seleccionó el tiempo empleado cuando era desplazamiento activo. Con un carácter cuantitativo b) Practica de AF no estructurada: donde se preguntó el volumen en minutos/semana de AF como jugar en el patio, en el parque, plaza, etc. donde se diferenció entre el volumen desempeñado entre semana y en el fin de semana y, c) Práctica de AF estructurada: donde se contempló el volumen en minutos/semana en actividades extraescolares diferenciando a su vez entre el tiempo empleado entre semana y en el fin de semana. Una vez obtenida esta información, se sumaron los minutos totales de AF diferenciando entre dos momentos: entre semana (lunes a viernes), y fin de semana (sábado y domingo). Todas estas preguntas han sido anteriormente empleadas por diferentes autores para conocer la práctica de actividad física en edades escolares (Cancela-Carral et al., 2016; Chillón et al., 2017; Muñoz-Galiano et al., 2019).

\section{Procedimiento}

Las pruebas que se describen en el sub-epígrafe anterior se llevaron a cabo en una semana de rutina escolar común, sin días festivos, días de actividad de centro, etc. Los instrumentos se rellenaron en un lugar habilitado para tal efecto donde se pudieron resolver las dudas.

Para el análisis estadístico se utilizó el paquete estadístico SPSS versión 24.0 para Windows. Se realizó un análisis descriptivo de los datos mostrados como media y desviación típica. Se confirmó una distribución no paramétrica por medio de la prueba Kolmogorov-Smirnov. Se empleó la prueba de Kruskal-Wallis para observar las diferencias entre el nivel educativo (bajo, medio y alto) atendiendo a la prueba post hoc ajustada al criterio ajustado de Bonferroni para la comparación por pares. Por medio de la prueba U-Mann-Whitney se analizaron las diferencias entre igualdad o no en el nivel educativo por parte de los progenitores (igual vs diferente nivel educativo). La significación se fijó en $p<0,05$.

\section{Resultados}

En la tabla 1 se muestran los resultados respecto al volumen de actividad física. 
Orientación familiar y hábitos saludables en estudiantes de Educación... Inés María Muñoz et al.

\section{Tabla 1}

Diferencias del volumen de práctica de actividad física según el nivel educativo de los progenitores

\begin{tabular}{|c|c|c|c|c|c|c|}
\hline & $\begin{array}{l}\text { Bajo Nivel } \\
\text { Educativo } \\
(n=136)\end{array}$ & $\begin{array}{l}\text { Medio Nivel } \\
\text { Educativo } \\
(n=107)\end{array}$ & $\begin{array}{l}\text { Alto Nivel } \\
\text { Educativo } \\
(n=41)\end{array}$ & $\begin{array}{l}\text { Total } \\
(n=284)\end{array}$ & $\begin{array}{l}\text { Chi- } \\
\text { Cuadrado }\end{array}$ & $\mathbf{P}$ \\
\hline $\begin{array}{l}\text { Tiempo Actividad } \\
\text { Física entre } \\
\text { semana (min) }\end{array}$ & $225,6 \pm 157,8$ & $442,8 \pm 307,2$ & $433,2 \pm 306,6$ & $337,2 \pm 123,0$ & 18,527 & $p=0,000$ \\
\hline $\begin{array}{l}\text { Tiempo Actividad } \\
\text { Física fin de } \\
\text { semana (min) }\end{array}$ & $96,6 \pm 48,6$ & $177,6 \pm 162,0$ & $219,0 \pm 121,2$ & $144,6 \pm 92,4$ & 15,072 & $p=0,001$ \\
\hline $\begin{array}{l}\text { Total, Actividad } \\
\text { Física (min) }\end{array}$ & $322,8 \pm 256,8$ & $620,4 \pm 482,4$ & $652,8 \pm 497,4$ & $482,4 \pm 387,6$ & 19,916 & $p=0,000$ \\
\hline Total, AF (min/día) & 46,11 & 88,62 & 68,91 & 80,4 & & \\
\hline $\begin{array}{l}\text { Cumplimiento } \\
\text { recomendación } \\
\text { salud ( } 60 \mathrm{~min} / \text { día) }\end{array}$ & $76,85 \%$ & $147,7 \%$ & $114,85 \%$ & $134 \%$ & & \\
\hline
\end{tabular}

Fuente: Elaboración propia

Se observan diferencias estadísticamente significativas fundamentalmente en las familias donde existe un nivel educativo medio o alto, respecto a las familias con bajo nivel educativo $(p<0,001)$. Existen diferencias significativas en la AF en el fin de semana donde el volumen es mayor en el nivel educativo Alto versus Medio. Por otro lado, teniendo en cuenta los datos generales de cumplimiento de AF, se observa como los niños/as del grupo de progenitores con medio nivel educativo y alto, cumplen con las recomendaciones de 60 minutos/día de AF, mientras que el grupo de bajo nivel educativo no. En la tabla 2, se observa la influencia del volumen de práctica en relación con si las familias poseen el mismo nivel educativo o diferente entre ambos progenitores (o padres/madres).

\section{Tabla 2}

Diferencias entre igualdad de nivel educativo de los progenitores y el volumen de práctica de actividad física

\begin{tabular}{lllll}
\hline & $\begin{array}{l}\text { Igual Nivel Educativo } \\
(\mathbf{n}=154)\end{array}$ & $\begin{array}{l}\text { Diferente } \\
\text { Educativo } \\
(\mathbf{n}=\mathbf{1 1 3})\end{array}$ & Nivel & $\mathbf{P}$ \\
\hline $\begin{array}{l}\text { Tiempo Actividad Física } \\
\text { entre semana (min) }\end{array}$ & $251,4 \pm 158,4$ & $488,4 \pm 409,8$ & $\mathrm{p}=0,001$ & $-3,318$ \\
$\begin{array}{l}\text { Tiempo Actividad Física } \\
\text { fin de semana (min) }\end{array}$ & $105,6 \pm 88,8$ & & & \\
$\begin{array}{l}\text { Total, Actividad Física } \\
\text { (min) }\end{array}$ & $357,0 \pm 268,8$ & $207,6 \pm 151,2$ & $\mathrm{p}=0,001$ & $-3,417$ \\
\hline
\end{tabular}

Fuente: Elaboración propia 
Los resultados se ofrecen de un total de 267 familias, ya que, de los participantes, diecisiete eran familias monoparentales, las cuales fueron anuladas de este apartado. Los datos indican diferencias altamente significativas, donde las parejas que poseen diferente nivel educativo entre ellas realizan mayor volumen de actividad física, tanto entre semana como en fin de semana $(p<0,001)$.

\section{Conclusiones y Discusión}

El nivel educativo de la familia influye en el volumen de práctica de actividad física en los menores de 6-11 años. De hecho, se observan diferencias estadísticamente significativas fundamentalmente en familias donde el nivel educativo es medio o alto respecto a las familias con bajo nivel educativo. A su vez, los hijos/as de progenitores con un nivel educativo medio/alto, son los únicos que cumplen con las recomendaciones saludable de AF diaria.

Estos datos ponen en relieve la importancia de orientar a las familias, especialmente en aquellas con bajo nivel educativo, las capacidades y habilidades necesarias para promocionar hábitos saludables en el contexto familiar. Igualmente, los resultados son de interés para los centros educativos a fin de implicar a las familias en programas de promoción de la actividad física.

La familia ejerce gran influencia en el desarrollo de hábitos y conductas de los menores, especialmente en la adquisición de estilos de vida saludables y la realización de actividad física (Kimiecik y Horn, 2012; Marques et al., 2014; Ramos y González, 2017; Suárez y Vélez, 2018). En esta línea, existen una serie de elementos vinculados a la familia que tienen algún grado de relación con el proceso educativo y el desarrollo de los hijos (López y Guimaro, 2015; Parra et al., 2017), entre los que se encuentra el nivel educativo de los progenitores.

A pesar de los estudios contradictorios en relación con la influencia de esta variable en la realización de actividad física (Lampinen et al., 2017; Lu et al., 2017; Torrijos et al., 2015; VidalConti, 2016), los resultados obtenidos en el presente estudio muestran que el nivel educativo de los progenitores influye significativamente en el tiempo de actividad física realizada por los niños de 6-11 años. Los mismos, coinciden con otros investigadores (Cheung, 2017; Lampinen et al., 2017; Muñoz-Galiano et al., 2019; Vidal-Conti, 2016).

Además, el tiempo de actividad física de los niños de Educación Primaria procedentes de familias con un mayor nivel educativo, aumenta durante el fin de semana en los niños. Como exponen autores como Määttä et al. (2017), el nivel educativo de los padres se identifica como un indicador importante para el sistema socioeconómico de la familia, lo que explica diferencias en el entorno social y físico del hogar, así como una mayor comprensión sobre la importancia de adoptar hábitos de vida saludables cuando el nivel educativo de los padres es medio-alto. Esto podría justificar que los niveles de actividad física de los niños de familias con medio-alto nivel educativo aumenten durante este periodo en comparación con aquellos cuyos progenitores tienen niveles educativos más bajos. Las creencias de los padres, sus niveles de actividad física, su apoyo a los hijos en prácticas deportivas, o la realización de actividades comunes, son considerados factores importantes para el desarrollo de actividad física y estilos de vida saludables (Kimiecik y Horn, 2012; Yao y Rhodes, 2015). De hecho, los menores cuyos progenitores tienen un nivel educativo medio-alto, son los que cumplen las recomendaciones de la 
OMS (2010) respecto a la realización de actividad física diaria (60 minutos de actividad física moderada a vigorosa diariamente).

Otro aporte interesante de este artículo es lo referente a la influencia del igual o diferente nivel educativo de los progenitores. En este sentido, diferente nivel educativo dio como resultado una mayor práctica de AF en todos los momentos evaluados. Esto es importante, porque existen estudios que indican que un mayor nivel educativo de la madre implica que los hijos/as hagan más AF (Gordon-Larsen et al., 2000; Huppertz et al., 2016; Román et al., 2006), a diferencia de otros, que indican que esta circunstancia se da cuando son los padres los que obtienen mayor nivel educativo (Kantomaa et al., 2007; Palou et al., 2005). Es cierto que, en este estudio, solo se ha evaluado si la pareja tiene igual o diferente nivel educativo y no qué miembro de la pareja tiene mayor o menor nivel, aunque recientemente en población infantil no se han observado diferencias a este respecto (Muñoz-Galiano et al., 2019), con lo que, quizás, haya una influencia más asociada para la orientación familiar y la autonomía que van adquiriendo los hijos/as según avanzan en edad.

Este estudio tiene a su vez, algunas limitaciones. La información ha sido proporcionada por medio de cuestionarios, lo que implica poder tener un resultado con una influencia de la percepción subjetiva del sujeto. Aunque los resultados son interesantes y abren una prospectiva de futuro, es cierto que se ha recogido de un solo centro educativo, lo que implica tomar con precaución los resultados. No obstante, este estudio aporta cómo el nivel educativo de los progenitores es un factor que considerar en el desarrollo de prácticas que ayuden a la familia a evitar hábitos poco saludables y promocionar la actividad física de sus hijos e hijas.

La familia tiene un importante papel en la tarea socioeducativa de sus hijos, a lo que habría que añadir, que esta función no solo abarca a la familia, sino que "es el grupo social con mayor grado de influencia en la evolución de la sociedad en su conjunto" (Martínez y Álvarez, 2002, p. 7). En este sentido, a pesar de que la familia, la vida familiar y los hábitos que en este contexto se adquieren reflejan los cambios de la sociedad, también se debe considerar la incidencia que ésta tiene en la sociedad (Mazo et al., 2019; Perea, 2006; Suárez y Vélez, 2018), lo que vendría a enfatizar la proyección social de la orientación familiar (Corchuelo et al., 2019; Martínez-Otero, 2018). Desde esta perspectiva, y de acuerdo con los resultados, la orientación familiar ha de contemplar la necesidad de la familia con menor nivel educativo de una mayor formación e información que le permita abordar su tarea educativa y social con eficacia, a fin de conseguir hábitos de vida saludables. En este sentido, algunas aplicaciones prácticas una vez que el centro educativo tiene información sobre la familia, serían: generar programas de información y divulgación de la importancia de la AF como eje saludable y diseñar programas en los que confluyan educación y actividad física desde la orientación familiar.

\section{Referencias bibliográficas}

Acuña-Collado, V. (2016). Family and school: crisis of participation in contexts of vulnerability. Revista Brasileira de Estudios Pedagógicos, 97(246), 255-272. http://dx.doi.org/10.1590/S2176-6681/267830331

Aguirre, A.M. (2015). Orientación familiar. En A.M. Aguirre, C. Caro, S. Fernández y M. Silvero (Coords.), Familia, escuela y sociedad. Manual para maestros (pp. 157-176). UNIR. 
Orientación familiar y hábitos saludables en estudiantes de Educación... Inés María Muñoz et al.

Álvarez González, M. y Bisquerra Alzina, R. (2012). Orientación educativa. Modelos, áreas, estrategias y recursos. Wolters Kluwer.

Azpillaga, V., Intxausti, N. y Joaristi, L. (2014). Implicación de las familias en los centros escolares de alta eficacia en la Comunidad Autónoma Vasca. Bordón, 66(3), 27-37. http://hdl.handle.net/11162/105065

Bagordo, F., De Donno, A., Grassi, T., Guido, M., Devoti, G., Ceretti, E., Zani, C., Feretti, D., Villarini, M., Moretti, M., Salvatori, T., CArducci, A., Verani, M., Casini, B., Bonetta, S., Carraro, E., SChiliró, T., Bonizzoni, S., Bonetti, A., Gelatti, U. y MAPEC LIFE study group (2017). Lifestyles and socio-cultural factors among children aged 6-8 years from five Italian towns: the MAPEC_LIFE study cohort. BMC Public Health, 17(233), 1-12. http://doi.org/10.1186/s12889-017-4142-x

Bernard, O. y LLevot, N. (2015). La participación de las familias en la escuela: factores clave. Revista de la Asociación de Sociología de la Educación (RASE), 8(1), 2.

Biddle, S.J. y Asare, M. (2011). Physical activity and mental health in children and adolescents: A review of reviews. British Journal of Sports Medicine, 45(11), 886-888. http://dx.doi.org/10.1136/bjsports-2011-090185

Cano, R. y Casado, M. (2015). Escuela y familia. Dos pilares fundamentales para unas buenas prácticas de orientación educativa a través de la escuela de padres. Revista Electrónica Interuniversitaria de Formación del Profesorado, 18(2), 15-27. http://dx.doi.org/10.6018/reifop.18.2.219491

Cancela-Carral, J.M., Lago-Ballesteros, J., Ayan-Perez, C. y Mosquera, M.B. (2016). Análisis de validez y fiabilidad de tres cuestionarios de autoinforme para valorar la actividad física realizada por adolescentes españoles. Gaceta Sanitaria, 30(5), 333-338. http://dx.doi.org/10.1016/i.gaceta.2016.04.009

Cheung, P. (2017). Children's after-school physical activity participation in Hong Kong: Does family socioeconomic status matter? Health Education Journal, 76(2), 221-230. https://doi.org/10.1177/0017896916660863

Chillón, P., Herrador-Colmenero, M., Migueles, J., Cabanas-Sánchez, V., Fernandez-Santos, J., Veiga, O. y Castro-Piñero, J. (2017). Convergent validation of a questionnaire to assess the mode and frequency of commuting to and from school. Scandinavian Journal of Public Health, 45, 612-620. https://doi.org/10.1177/1403494817718905

Chillón, P., Ortega, F.B., Ferrando, J.A. y Casajus, J.A. (2011). Physical fitness in rural and urban children and adolescents from Spain. Journal Science and Medicine Sport, 14, 417-423. https://doi.org/10.1016/j.jsams.2011.04.004

Corchuelo, C., Cejudo, C.M.A. y Tirado, R. (2019). Las conexiones entre apoyo familiar, escolar y el compromiso de los estudiantes en un centro de compensación educativa. Revista Española de Orientación y Psicopedagogía, 30(2), 46-52. https://doi.org/10.5944/reop.vol.30.num.2.2019.25337

Cornejo, R. y Redondo, J. M. (2007). Variables y factores asociados al aprendizaje escolar: una discusión desde la investigación actual. Estudios pedagógicos, 33(2), 155-175.

Cuervo, C., Cachón, J., González, C. y Zagalaz, M.L. (2017). Hábitos alimentarios y práctica de actividad física en adolescentes. Journal of Sport and Health Research. 9(1), 75-84.

De la Torre-Cruz, M. J., Ruiz-Ariza, A., López-García, M. D., y Martínez-López, E. J. (2015). Efecto diferencial del estilo educativo materno y paterno sobre el autoconcepto físico del 
Orientación familiar y hábitos saludables en estudiantes de Educación... Inés María Muñoz et al.

adolescente. Revista de Educación, 369, 59-84. http://doi.org/10.4438/1988-592X-RE2015-369-290

Drenowatz, C., Eisenmann, J., Pfeiffer, K., Welk, G., Heelan, K., Gentile, S. y Walsh, D. (2010). Influence of socio-economic status on habitual physical activity and sedentary behavior in 8- to 11-year-old children. BMC Public Health, 10, 214. https://doi.org/10.1186/1471-2458$\underline{10-214}$

Dumith, S. C., Hallal, P. C., Reis, R. S. y Kohl, H. W. (2011). Worldwide Prevalence of Physical Inactivity and its Association with Human Development Index in 76 Countries. Preventive Medicine, 53, 24-28.

Edwarson, C.L. y Gorely, T. (2010). Parental influences on different types and intensities of physical activity in youth: a systematic review. Psychology of Sport and Exercise, 11, 522535. https://doi.org/10.1016/i.psychsport.2010.05.001

Fajardo-Bullón, F., Maestre Campos, M., Felipe Castaño, E., León del Barco, B. y Polo del Río, M.I. (2017). Análisis del rendimiento académico de los alumnos de Educación Secundaria Obliga-toria según las variables familiares. Educación XXI, 20(1), 209-232. http://doi.org/10.5944/educxx1.17509

Gomáriz, M.A., Parra, J., García-Sanz, M.P., Hernández, M.A. y Pérez, J. (2008). La comunicación entre la familia y el centro educativo. Consejo Escolar de la Región de Murcia. Consejería de Educación, Ciencia e Investigación.

González, O. (2014). Familia y escuela, escuela y familia. Desclée De Brovwer.

Gordon-Larsen, P., McMurray, R. y Popkin, B.M. (2000). Determinants of adolescent physical activity and inactivity patterns. Pediatrics, 105(6), e83-e83. https://doi.org/10.1542/peds.105.6.e83

Hayes, D. (2017). The love of sport: an investigation into the perceptions and experiences of physical education amongst primary school pupils. Research Papers in Education. 32(4), 518-534.

Huppertz, C, Bartels, M., De Geus, E.J. C., Van Beijsterveldt, C.E.M., Rose, R.J., Kapiro, J. y Silventoinen, K. (2016). The effects of parental education on exercise behavior in childhood and youth: a study in Dutch and Finnish twins. Sandinavian Journal of Medicine \& Science in Sports, 27(10), 1143-1156. https://doi.org/10.1111/sms.12727

Jalali-Farahani, S., Amiri, P., Abbasi, B., Karimi, M., Cheraghi, L., Daneshpur, A.S. y Azizi, F. (2017). Maternal characteristics and incidence of overweight obesity in children: a 13 year follow up study in an eastern Mediterranean population. Maternal Child Health Journal, 21(5), 1211-1220. https://doi.org/10.1007/s10995-016-2222-7

Kantomaa, M. T., Tammelin, T. H., Nayha, S. y Taanila, A.M. (2007). Adolescents' physical activiy inr elation to family income and parents' education. Preventive Medicine, 44(5), 410-415. https://doi.org/10.1016/i.ypmed.2007.01.008

Kimiecik, J. y Horn, T. (2012). Examining the relationship between family context and children's physical activity beliefs: the role of parenting style. Phychology of Sport and Exercise, 13, 10-18. https://doi.org/10.1016/i.psychsport.2011.08.004

Lampinen, E.K., Eloranta, A.M., Haapala, E., Lindi, V., Väistö, J., Lintu, N., Karjalainen, P., Kukkonen-Harjula, K., Laaksonen, D. y Lakka, T. (2017). Physical activiy, sedentary behaviour, and socioeconomic status among Finnish girls and boys aged 6-8 years. $\begin{array}{llll}\text { European Jorunal of Sports } & \text { Science, } & 17(4), & 462-472 .\end{array}$ https://doi.org/10.1080/17461391.2017.1294619 
Orientación familiar y hábitos saludables en estudiantes de Educación... Inés María Muñoz et al.

López, G. y Guaimaro, Y. (2015). El rol de la familia en los procesos de educación y desarrollo humano de los niños y niñas. Revista Universitaria de Desarrollo Social, 10, 31-55.

Lu, C., Stock, R.P., Sauer, P., Sijtsma, A., Wiersma, R., Huang, G. y Corpeleijn, E. (2017). Factors of physical activity among Chinese children and adolescents: a systematic review. International Journal of Behavioral Nutrition and Physical Activity, 14(36), 1-10. https://doi.org/10.1186/s12966-017-0486-y

Määttä, S., Kaukonen, R., Vepsalainene, H., Lehto, E., Ylonen, A., Ray, C., Erkkola, M. y Roos, E. (2017). The mediation role of the home environment in relation to parental educational level and preschool children's screen time: a cross-sectional study. BMC Public Health, 17, 688. https://doi.org/10.1186/s12889-017-4694-9

Marques, A., Martins, J., Sarmento, H., Diniz, J. y Carreiro da Costa, F. (2014). Adolescents 'physical activity profile according to parental physical activity participation. Journal of Human Sport \& Exercise, 9(1), 81-90

Martínez, M.C. y Álvarez, B. (2002). Orientación familiar. UNED.

Martínez-González, R.A., Rodríguez, B., y Gimeno, J. L. (2010). Áreas de cooperación entre los centros docentes y las familias. Estudio de caso. Educatio siglo XXI, 28(1), 127-156.

Martínez-Otero, V. (2018). Orientación personal y familiar. Editorial CCS.

Mazo, Y.I., Mejía, L.A. y Muñoz, Y.P. (2019). Calidad de vida: la familia como una oportunidad transformadora. Paiésis, 36, 98-110. https://doi.org/10.21501/issn.1692-0945

Muñoz-Galiano, I., Connor, J.D., Gómez-Ruano, M.A. y Torres-Luque, G. (2020). Influence of the parental educational level on physical activity in schoolchildren. Sustainability, 12, 3920. https://doi.org/10.3390/su12093920

Muñoz-Galiano, I., Hernández-García, R. y Torres-Luque, G. (2019). Influencia del nivel educativo del progenitor sobre la práctica de actividad física en estudiantes de Educación Infantil. Journal of Sport and Health Research, 11(2), 161-170.

Nogueira, M.A. y Ceinos, M.C. (2015). Influencia de las tablets en el desarrollo infantil: perspectivas y recomendaciones a tener en cuenta en la orientación familiar. Tendencias Pedagógicas, 26, 33-50.

OMS (2010). Recomendaciones mundiales sobre Actividad Física y Salud, Ginebra. Suiza, http://www.who.int/dietphysicalactivity/factshett recommendations/es/index.html

Palou, P., Ponseti, F., Gili, M., Borrás, P. y Vidal, J. (2005). Profile of athletic habits of preadolescents in Mallorca. Revista de Psicología del Deporte, 14(2), 225-236.

Pàmies-Rovira, J., Senent-Sánchez, J.M. y Essomba-Gelabert, M. A. (2016). El liderazgo pedagógico y la implicación del profesorado como factores de éxito en centros de entornos $\begin{array}{llll}\text { desfavorecidos en } \quad \text { España. } & \text { RELIEVE, }\end{array}$ http://dx.doi.org/10.7203/relieve.22.2.7600

Parra, J., Gomariz, M.A., Hernández-Prados, M.A. y García-Sanz, M.P. (2017). La participación de las familias en Educación Infantil. RELIEVE, 23(1), 1-26. http://doi.org/10.7203/relieve.23.1.9258

Perea, R. (2006). La familia como contexto para un desarrollo saludable. Revista Española de Pedagogía, año LXIV (235), 417-428. 
Orientación familiar y hábitos saludables en estudiantes de Educación... Inés María Muñoz et al.

Pérez-Bóveda, A. y Yáñiz, C. (2017). Programas de formación parental. Análisis comparativo. Revista Española de Orientación y Psicopedagogía, 26(2), 104-122. http://www.redalyc.org/articulo.oa?id=338241632008

Ramos, Y. y González, M.A. (2017). Un acercamiento a la función educativa de la familia. Revista Cubana de Medicina General Integral, 33(1), 100-114.

Ramírez-García, A., Salcines-Tallado, I. y González-Fernández, N. (2020). Los dispositivos móviles en el hogar. Interés formativo de las familias españolas. Revista Española de

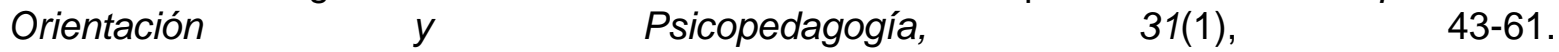
https://doi.org/10.5944/reop.vol.31.num.1.2020.27286

Román, B., Serra, LL., Ribas, L, Pérez-Rodrigo, C. y Aranceta, J. (2006). Physical activiy in Spanish youth's in free time. Apunts: Medicina de I'Esport, 41(151), 86-94.

Romero-Blanco, C., Dorado-Suárez, A., Jiménez-Zazo, F., Castro-Lemus, N. y Aznar, S. (2020). School and family environment is positively associated with extracurricular physical activity practice among 8 to 16 years ald schoolboys and girls. International Journal of Environmental Research and Public Health, 17(15), 5371. https://doi.org/10.3390/ijerph17155371

Ruiz-Ariza, A., Ruiz, J.R., De la Torre-Cruz, M.J., Latorre-Román, P. y Martínez-López, E.J. (2016). Influencia del nivel de atracción hacia la actividad física en el rendimiento académico de los adolescentes. Revista Latinoamericana de Psicología, 48, 42-50. https://doi.org/10.1016/i.rlp.2015.09.005

Sawa, S., Sekine, M., Yamada, M., Fukazawa y Hiraku, Y. (2020). Social and family factors as determinants of exercise habits in Japanese elementary school children: a cross-sectional study from the Super Shokuiku School Project. Environmental Health and Preventive Medicine, 25, 54. https://doi.org/10.1186/s12199-020-00892-3

Sheldon, S.B. y Epstein, J.L. (2002). Improving student behavior and school discipline with family and community involvement. Education and Urban Society, 35(1), 4-26. https://doi.org/10.1177/001312402237212

Silvero, M. (2015). La educación familiar en un mundo de cambio. En A.M. Aguirre, C. Caro, S. Fernández y M. Silvero (Coords.), Familia, escuela y sociedad. Manual para maestros (pp. 31-54). UNIR.

Siles-Rojas, C. (2003). La colaboración de los padres con la escuela. Revista Padres y Maestros, PyM, 279, 10-14.

Sousa, G. y Silva, D. (2017). Sedentary behavior based on screen time: prevalence and associated sociodemographic factor in adolescents. Ciência \& Saúce Colectiva, 22(2), 4061-4072. https://doi.org/10.1590/1413-812320172212.00472016

Suárez, P. y Vélez, M. (2018). El papel de la familia en el desarrollo social del niño: una mirada desde la afectividad, la comunicación familiar y estilos de educación parental. Revista Psicoespacios, 12(20), 153-172. http://doi.org/10.25057/issn.2145-2776

Torres, J., Contreras, S., Lippi, L., Huaiquimilla, M. y Leal, R. (2019). Hábitos de vida saludable como indicador de desarrollo personal y social: discursos y prácticas en escuelas. Calidad en la educación (50), 357-392. http://dx.doi.org/10.31619/caledu.n50.728

Torrijos, C., Pardo, M.J., Solera, M., Gulías, R., Amador, S. y Arias, N.M. (2015). Relación entre nivel de estudios de los padres y condición física de los escolares. Diferencias de género. Revista Andaluza de Medicina del Deporte, 8(1), 20-47. 
Orientación familiar y hábitos saludables en estudiantes de Educación... Inés María Muñoz et al.

Vidal-Conti, J. (2016). Identification of physical activity predictors in schoolchildren according to a socio-ecological model using multifactorial analysis. Cultura, Ciencia y Deporte, 11(31), 5159.

Wu, S., Bastian, K., Hinmaa, A. y Veugelers, P. (2018). Influence of physical activity, sedentary behaviour, and diet quality in childhood on the incidence of internalizing and externalizing disorders during adolescence: a population-based cohort study. Annals of Epidemiology, 28, 86-94. https://doi.org/10.1016/j.annepidem.2017.12.002

Yao, Ch. y Rhodes, R. (2015). Parental correlates in child and adolescent physical activity: a metaanalysis. International Journal of Behavioral Nutrition and Physical Activity, 12,10. https://doi.org/10.1186/s12966-015-0163-y

Fecha de entrada: 21 junio 2020

Fecha de revisión: 6 noviembre 2020

Fecha de aceptación: 23 diciembre 2020 